\title{
Comparing the Effects of Network Latency versus Local Latency on Competitive First Person Shooter Game Players
}

\author{
Shengmei Liu, Mark Claypool \\ sliu7,claypool@wpi.edu \\ Worcester Polytechnic Institute, Worcester, MA, USA
}

\author{
Atsuo Kuwahara, James Scovell, Jamie Sherman \\ atsuo.kuwahara,james.j.scovell,jamie.sherman@intel.com \\ Intel Corporation, Hillsboro, OR,, USA
}

\begin{abstract}
Competitive gamers, and especially esports gamers, want lower latency to improve their chances of winning. There are two main sources of latency in competitive computer games: local system latency and network latency. While researchers have studied the effects of both, a direct comparison has not been done, especially for competitive gamers and low-end latencies. This paper assesses the effects of local latency and network latency on experienced Counter-strike: Global Offensive players comparing data from two user studies. Analysis of the results shows that local latency has about a 2x higher impact on player performance (accuracy and score) and Quality of Experience (QoE) than does the same amount of network latency.
\end{abstract}

\section{CCS CONCEPTS}

-Applied computing $\rightarrow$ Computer games; $\bullet$ Human-centered computing $\rightarrow$ User studies.

\section{KEYWORDS}

skill, gamer, FPS, user study, esports, lag, CS:GO

\section{ACM Reference Format:}

Shengmei Liu, Mark Claypool and Atsuo Kuwahara, James Scovell, Jamie Sherman. 2018. Comparing the Effects of Network Latency versus Local Latency on Competitive First Person Shooter Game Players. In EHPHCI '21: ACM Esports and High Performance HCI, May 8, 2021, Virtual Conference. ACM, New York, NY, USA, 5 pages. https://doi.org/10.1145/1122445.1122456

\section{INTRODUCTION}

Computer games are one of the world's most popular forms of entertainment, with global sales increasing at an annual rate of $10 \%$ or more [19]. Esports, in particular, are popular and lucrative - the largest esports prize pools are about $\$ 25$ million USD [7], and by 2023, there are expected to be about 300 million frequent viewers of esports worldwide, an increase from 173 million in 2018 [9].

Latency between a player's input and the esports game output can impact the responsiveness and consistency of the game, hurting player performance and degrading quality of experience. There are two main sources of latency in esports games - from the local system, such as from the mouse, OS and monitor, and from the

Permission to make digital or hard copies of all or part of this work for personal or classroom use is granted without fee provided that copies are not made or distributed for profit or commercial advantage and that copies bear this notice and the full citation on the first page. Copyrights for components of this work owned by others than ACM must be honored. Abstracting with credit is permitted. To copy otherwise, or republish, to post on servers or to redistribute to lists, requires prior specific permission and/or a fee. Request permissions from permissions@acm.org.

EHPHCI '21, May 8, 2021, Virtual Conference

(C) 2018 Association for Computing Machinery.

ACM ISBN 978-1-4503-XXXX-X/18/06 ..\$15.00

https://doi.org/10.1145/1122445.1122456 network between the client and the server. While both sources of latency affect the player, they manifest differently - local latency lags all player input until game output, while network latency lags communication to the server. This means local latency makes game controls feel unresponsive, while network latency makes player actions resolved later by the server. While competitive gamers typically seek to reduce latency or mitigate its effects - the conventional wisdom being "faster is better" - the extent to which both types of latency affect players remains unknown.

There have been studies on network latency and commercial games $[8,10]$, especially latency and first-person shooter (FPS) games $[1-3,18]$ owing to the sensitivity of FPS games to network latency and the prevalence of FPS games in the competitive and esports scenes. However, such studies often evaluate non-expert gamers or high-end latencies (e.g., above $150 \mathrm{~ms}$ ) that are not typically seen by competitive gamers. Other games research has studied local latency, usually focusing on a subset of a full game $[12,15,16]$. While this prior research has been valuable for understanding latency and games, to the best of our knowledge, there has been no direct comparison between local latency and network latency for competitive game players.

This paper compares the effects of network latency and local latency using data from two user studies that measure the impact of latency on experienced FPS game players. For both studies, players skilled at the esports FPS game Counter-strike: Global Offensive (CS:GO) (Valve, 2012) played rounds of CS:GO with controlled amounts of network latency or local latency. A total of sixty-eight (68) participants across both studies provide performance (accuracy, score) and opinions ( $\mathrm{QoE})$ that are used to assess and compare the impact of different types and amounts of latency.

Analysis of the results shows that from a baseline of 125 milliseconds of total latency, reducing local latency by 100 milliseconds results in an improvement of 6 percent for accuracy, 3 points per minute for score and 1.6 points (on a 5 point scale) for QoE, while reducing network latency by 100 milliseconds results in an improvement of 2 percent for accuracy, 2 points per minute for score and 0.7 points for QoE. In short, local latency has about twice the impact as network latency for competitive FPS game players.

The rest of this paper is organized as follows: Section 2 describes related work; Section 3 summarizes the user studies; Section 4 describes the results; Section 5 analyzes and compares local latency to network latency; and Section 6 summarizes our conclusions and presents possible future work.

\section{RELATED WORK}

For local latency, Ivkovic et al. [11] find significant main effects for local latency on target tracking and acquisition tasks with a greater effect for higher target speeds. Long and Gutwin [15] find target 
speed affects how much local latency increases target acquisition time, with fast targets affected by latencies as low as $50 \mathrm{~ms}$ but slower targets resilient to latencies as high as $150 \mathrm{~ms}$. Long and Gutwin [16] also compare the effects of local latency on moving target selection across 4 different gaming devices, demonstrating that latency affects each device differently. Claypool et al. [4] show local latency and target speed exponentially impact target selection time. Spjut et al. [21] show reduced local latencies have a clear benefit in a first person targeting task, more so than does a higher frame rate display.

For network latency and FPS games, Armitage et al. [2] estimate the network latency tolerance threshold for Quake 3 to be about 150-180 ms. Dick et al. [6] show players find $150 \mathrm{~ms}$ acceptable for Counter-strike and Unreal Tournament 2003. Quax et al. [18] find UT2003 players suffer with latency and jitter as low as $100 \mathrm{~ms}$. Amin et al. [1] demonstrate player experience determines network latency sensitivity for Call of Duty, with competitive gamers more adept at compensating for impaired network conditions. For network latency and other game genres, Fritsch et al. [8] find players of the role-playing game Everquest 2 can tolerate hundreds of milliseconds of network latency. Hoßfeld et al. [10] show players of the casual game Minecraft are insensitive to network latencies of up to 1 second. Sheldon et al. [20] find some aspects of play in the real-time strategy game Warcraft 3 are not affected by up to a second of network latency.

While useful for understanding the effects of local latencies and network latencies on games, in contrast to our work, these works do not compare local latency to network latency, nor do they necessarily represent competitive FPS player performance.

\section{USER STUDIES}

We use data obtained from two user studies, one focusing on local latency [13] and the other on network latency [14]. The studies themselves were not designed to be directly comparable, but the settings and methods are similar enough to allow for comparative analysis. Each dataset was obtained from users playing Counter strike: Global Offensive (Valve, 2012) with the automatic AK-47 rifle on the small Mirage map in short, free-for-all rounds with $20 \mathrm{AI}-$ controlled bots. The local latency study added a different latency each round $(0,25,50,75$, or $100 \mathrm{~ms})$, delaying the mouse and the keyboard with a custom tool called evlag. The network latency study also added a different latency each round $(25,50,100$, or 150 $\mathrm{ms}$ ), but delayed the client-server communication with the network tool clumsy, ${ }^{1}$ on top of $<1$ ms of LAN latency.

For the PC's used in both studies, base local latency was measured the same way: using a high-framerate camera to capture and count the frames between the moment a user presses the mouse button and the resulting screen output, repeated 10 times. The local latency study base was measured at $M=25.2 \mathrm{~ms}, S D=2.8 \mathrm{~ms}$ and the network latency study base was $M=24.6 \mathrm{~ms}, S D=3.44 \mathrm{~ms}$. Hereafter in this paper, $25 \mathrm{~ms}$ is added to all latency analysis.

For both studies, users were screened to ensure a high-level of skill in CS:GO - a minimum of 100 hours of previous CS:GO play was required in order to participate.

\footnotetext{
${ }^{1}$ https://jagt.github.io/clumsy/
}

Table 1: Subjective questions per round

\begin{tabular}{lll}
\hline & Rate (low 1 to 5 high): & Source \\
\hline Q1 & The responsiveness of the round & Long [16] \\
Q2 & Your annoyance with the unresponsiveness & GEQ [17] \\
Q3 & Your frustration in the round & iGEQ [17] \\
Q4 & How much your performance was due to you & Attribution [5] \\
\hline
\end{tabular}

Table 2: Demographics

\begin{tabular}{ccccc}
\hline Latency & Users & Age (yrs) & Gender & FPS Skill \\
\hline Local & 43 & $21.2(5.0)$ & $42 \sigma^{7}, 1$ 우 & $4.5(0.7)$ \\
Network & 25 & $20.8(3.0)$ & $25 \sigma^{7}, 0$ 우 & $4.4(0.7)$ \\
\hline
\end{tabular}

There was no upper limit on player score - the round ended after 4 minutes in the local latency study and 3.5 minutes in the network latency study. After each round, users filled out a subjective survey consisting of questions on a discrete 5-point Likert scale about the game experience in the preceding round. The questions in common for both studies are shown in Table 1 .

\section{RESULTS}

Forty-three (43) users passed screening to participate in the local latency user study, and twenty-five (25) users passed screening to participate in the network latency user study. Table 2 summarizes the participant demographics. FPS self-rating skill is on a five-point scale, 1 (low) to 5 (high). For age and FPS self-rating skill the mean values are given with standard deviations in parentheses.

Figure 1a depicts boxplot distributions for self-reported hours of CS:GO play. The boxes depict quartiles and medians for the distributions. Points higher or lower than $1.4 \times$ the inter-quartile range are outliers, shown by red pluses. The whiskers span from the minimum non-outlier to the maximum non-outlier. The black pluses show the mean values. The gray box represents the local latency study and the blue box the network latency study. Users in the local latency study average 664 hours of CS:GO play compared to an average of 832 hours in the network latency study. Figure 1 depicts self reported CS:GO skills. The points are mean values with error bars denoting standard deviations. Black is for the local latency study and blue for the network latency study. Based on Figure 1a and Figure 1, while both groups are experienced CS:GO players, the users in the network latency study are somewhat more experienced and skilled at CS:GO than the users in the local latency study.

This difference in skill is born out via aggregate performance in the studies. We measure user performance in terms of accuracy (shots hit divided by shots fired, reported as a percent) and game score (in CS:GO, score $=2 \times k i l l s+$ assists, reported as points per minute). The CS:GO log files are mined to determine number of hits, kills and assists by each user for each round, and the evlag log files provide the shots fired based on the number of left mouse-button clicks. Table 3 gives the mean weapon accuracy and score from both studies, with standard deviations in parenthesis. 


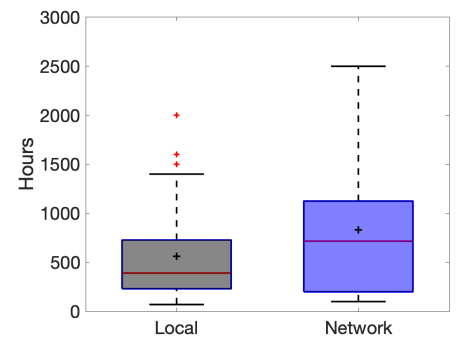

(a) Hours played

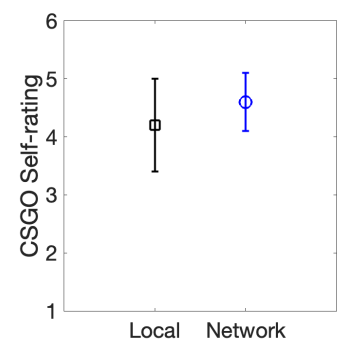

(b) Skill (1-low to 5-high)
Figure 1: Self-reporting for CSGO

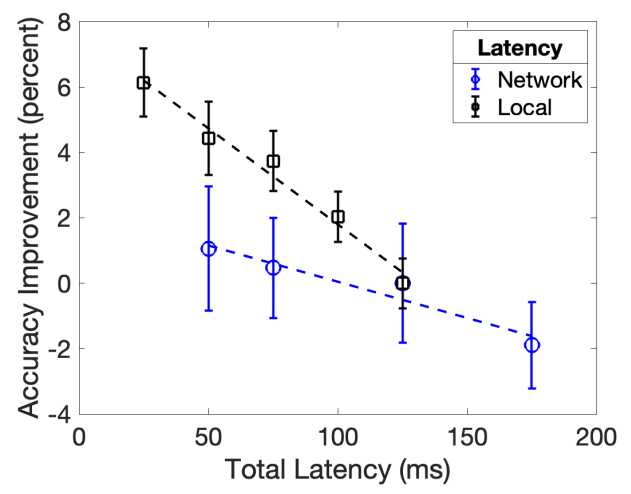

Figure 2: Accuracy improvement

Given the self-reported and observed difference in CS:GO skill between the two groups, for the rest of this paper, we do not directly compare user performance for each group but instead compare the relative impact of latency on performance.

\section{Table 3: User performance summary}

\begin{tabular}{lcc}
\hline Latency & Accuracy (\%) & Score $(\mathrm{pts} / \mathrm{min})$ \\
\hline Local & $17.8(4.8)$ & $11.5(3.2)$ \\
Network & $19.6(4.3)$ & $14.5(3.8)$ \\
\hline
\end{tabular}

\section{ANALYSIS}

This section compares user performance (Section 5.1) and Quality of Experience (Section 5.2) for network and local latencies.

\subsection{Performance}

Player performance is assessed by weapon accuracy (i.e., how readily a player hits an opponent with each shot) and game score (based on number of opponent kills and assists with kills).

5.1.1 Accuracy. Figure 2 depicts weapon accuracy versus latency. The $\mathrm{x}$ axis is the total latency, i.e., local latency plus network latency. The y axis is the weapon accuracy (percent) increase from the 125 ms total latency condition. For example, an accuracy of 15 percent at $125 \mathrm{~ms}$ of latency compared to an accuracy of 20 percent at 25

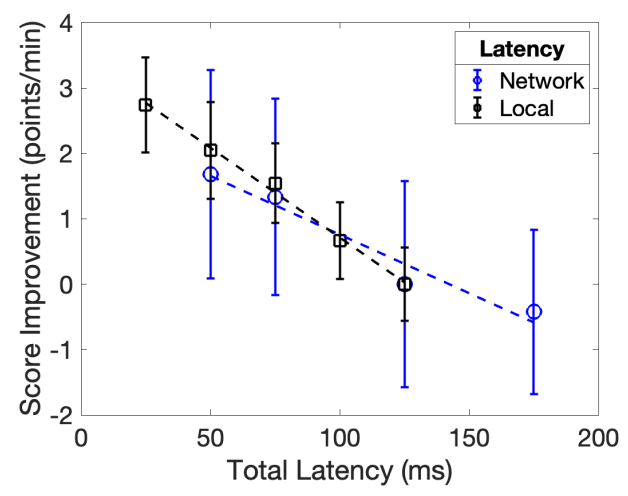

Figure 3: Score improvement

Table 4: Significance (compared to $125 \mathrm{~ms}$ )

\begin{tabular}{ccccc}
\multicolumn{6}{c}{ Local latency } \\
\hline Total & \multicolumn{3}{c}{ Accuracy } & \multicolumn{2}{c}{ Score } \\
$(\mathrm{ms})$ & $\mathrm{t}(42)$ & $\mathrm{p}$ & $\mathrm{t}(42)$ & $\mathrm{p}$ \\
\hline 25 & 14.23 & $<. \mathbf{0 0 1}$ & 8.56 & $<. \mathbf{0 0 1}$ \\
50 & 9.57 & $<. \mathbf{0 0 1}$ & 7.32 & $<. \mathbf{0 0 1}$ \\
75 & 9.86 & $<. \mathbf{0 0 1}$ & 5.13 & $<. \mathbf{0 0 1}$ \\
100 & 5.15 & $<. \mathbf{0 0 1}$ & 2.33 & $\mathbf{0 . 0 2}$ \\
\hline
\end{tabular}

\begin{tabular}{ccccc}
\multicolumn{4}{c}{ Network latency } \\
\hline Total & \multicolumn{3}{c}{ Accuracy } & \multicolumn{2}{c}{ Score } \\
$(\mathrm{ms})$ & $\mathrm{t}(24)$ & $\mathrm{p}$ & $\mathrm{t}(24)$ & $\mathrm{p}$ \\
\hline 50 & 1.20 & .24 & 2.35 & $\mathbf{. 0 3}$ \\
75 & 0.61 & .55 & 2.25 & $\mathbf{. 0 3}$ \\
175 & -2.77 & $\mathbf{. 0 1}$ & -0.92 & 0.37 \\
\hline
\end{tabular}

ms of latency would be a 5 percent improvement. The points are the means for all users for that latency condition, bounded by $95 \%$ confidence intervals. The dashed lines show a linear regression for the mean values. The blue points and lines denote the network latency study, and the black denotes the local latency study. The regressions fit the mean values well for both studies, with an $R^{2}$ of 0.98 and $p=.001$ for the local latency study, and an $R^{2}$ of 0.93 and $p=.038$ for the network latency study. Visually, the local latency slope is steeper than the network latency slope. As a take-away, a decrease in network latency by $100 \mathrm{~ms}$ improves player accuracy by an average of about 2 percent, while a decrease in the same amount of local latency improves player accuracy by an average of about 6 percent.

5.1.2 Score. Figure 3 depicts player score versus latency. The axes and points are as in Figure 2, but the data is the score $(2 \times$ kills + assists) improvement per minute instead of accuracy improvement. The regressions fit the mean values well for both studies, with an $R^{2}$ of 0.99 and $p<.001$ for the local latency study, and an $R^{2}$ of 0.96 and $p=.023$ for the network latency study. Again, visually, the slope for the local latency study is steeper than the slope for the network latency study. As a take-away, a decrease in local latency by $100 \mathrm{~ms}$ improves player score by about 3 points per minute of gameplay, while the same amount of decrease in network latency improves player score by 2 points per minute of gameplay. For reference, often less than a single point in a game separates the scores of top CS:GO players.

5.1.3 Effect Size. An effect size provides a measure of the magnitude of difference - in our case, the difference with the $125 \mathrm{~ms}$ total latency condition. We compare performance with latency to this condition by paired t-tests $(\alpha=0.05)$ for participants within each 
Table 5: Effect size (compared to $125 \mathrm{~ms}$ )

\begin{tabular}{|c|c|c|c|c|c|}
\hline \multicolumn{3}{|c|}{ Local latency } & \multicolumn{3}{|c|}{ Network latency } \\
\hline \multirow{2}{*}{$\begin{array}{l}\text { Total } \\
(\mathrm{ms})\end{array}$} & \multicolumn{2}{|c|}{ Effect size } & \multirow{2}{*}{$\begin{array}{l}\text { Total } \\
(\mathrm{ms})\end{array}$} & \multicolumn{2}{|c|}{ Effect size } \\
\hline & Accuracy & Score & & Accuracy & Score \\
\hline 25 & 1.57 & 0.94 & 50 & 0.24 & 0.47 \\
\hline 50 & 1.05 & 0.80 & 75 & 012 & 0.45 \\
\hline 75 & 1.08 & 0.57 & 15 & 0.12 & 0.45 \\
\hline 100 & 0.56 & 0.26 & $1 / 5$ & -0.53 & -0.18 \\
\hline
\end{tabular}

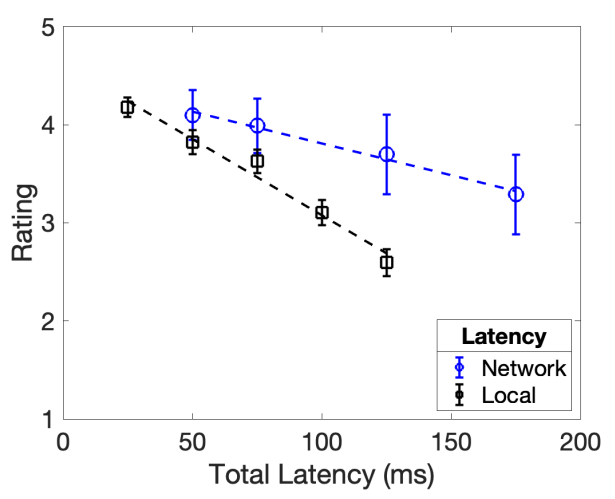

Figure 4: QoE

study and compute the Cohen's d effect sizes for the same. The Cohen's d effect size assesses the differences in means in relation to the pooled standard deviation. Generally, small effect sizes are anything under 0.2 , medium is 0.2 to 0.5 , large 0.5 to 0.8 , and very large above 0.8 . The results are shown in Table 4 and Table 5. For local latency, all the conditions are significant and reducing latency has a large effect on both accuracy and score, except for a medium effect at $100 \mathrm{~ms}$. For network latency, only the $175 \mathrm{~ms}$ condition is significant for accuracy and only 50 and $75 \mathrm{~ms}$ conditions are significant for score. Randomly sub-sampling 25 participants from the local latency study pool and re-computing significance (not shown) shows the lack of significance in the network latency study is only partly due to the smaller sample size $(N=25$ versus $N=43)$. Reducing network latencies from $125 \mathrm{~ms}$ has a small or at most medium effect for all conditions. As a take-away, the effects sizes for accuracy and score are larger for local latency (medium to large) than they are for network latency (small to medium).

\subsection{Quality of Experience}

Quality of Experience (QoE) is assessed from user responses to the 4 survey questions the studies have in common (see Table 1), filled out by users at the end of each round. Responses are a rating given on a discrete 5-point scale and, for the analysis, response data for question 2 and question 3 are rearranged so that a 1 is low (worse) and a 5 is high (better) for all questions.

For each individual question, QoE degrades with network latency and local latency - the linear regressions fit the means well for each question, with $R^{2}$ values from 0.901 to 0.999 .
For an overall measure of QoE, we compute the mean combined rating, weighting all questions equally. Figure 4 depicts the results. The $\mathrm{x}$ axis is the total latency in milliseconds and the $\mathrm{y}$ axis is the rating. The points are the means for all users for that latency condition, bounded by $95 \%$ confidence intervals. The dashed lines are linear regression fits through the mean values. The blue represents the network latency study and the black the local latency study. The linear regressions fit the means well for both studies, with $R^{2}$ 0.97 and $p=.002$ for local latency and $R^{2} 0.99$ and $p=.007$ for network latency. As a take-away, a decrease in local latency by 100 $\mathrm{ms}$ improves QoE by about 1.6 points on a 5-point scale, while the same amount of decrease in network latency improves QoE by 0.7 points.

\subsection{Summary}

Table 6 summarizes the linear regressions from the above analysis in tabluar form. The regressions fit the mean values well and all results are significant. Slopes for the local latency regressions are about $2 \mathrm{x}$ steeper than the slopes for the network latency regressions for all cases.

Table 6: Linear regression

\begin{tabular}{lcccc}
\hline Metric & Latency & Slope & $R^{2}$ & P value \\
\hline Accuracy & Local & -0.059 & 0.98 & $\mathbf{. 0 0 1}$ \\
Accuracy & Network & -0.022 & 0.93 & $\mathbf{. 0 3 8}$ \\
Score & Local & -0.027 & 0.99 & $<.001$ \\
Score & Network & -0.017 & 0.96 & $\mathbf{. 0 2 3}$ \\
QoE & Local & -0.016 & 0.97 & $\mathbf{. 0 0 2}$ \\
QoE & Network & -0.006 & 0.99 & $\mathbf{. 0 0 7}$ \\
\hline
\end{tabular}

\section{CONCLUSION}

Based on results from 68 users playing over 60 hours of CS:GO under controlled latency conditions, local latency has more impact on competitive FPS game players than does the same amount of network latency. In general, for a baseline system with 125 total milliseconds of network latency and local system latency:

(1) A decrease in $100 \mathrm{~ms}$ of local latency improves accuracy by 6 percent, score by 3 points/minute and QoE by 1.6 points on a 5 -point scale.

(2) A decrease in $100 \mathrm{~ms}$ of network latency improves accuracy by 2 percent, score by 2 points/minute and QoE by 0.7 points on a 5 -point scale.

Future work may compare local latency and network latency on additional game aspects, such as a broader range of player skills (i.e., non-expert gamers), player versus player (rather than versus bots), other weapon types (e.g., sniper rifles), as well as other FPS games to confirm that our results generalize. Possible studies also include alternate esports game genres, such as Multi-player Online Battle Arena (MOBA) (e.g., DOTA 2, League of Legends) and Real-Time Strategy (RTS) (e.g., Starcraft). 


\section{REFERENCES}

[1] R. Amin, F. Jackson, J. Gilbert, J. Martin, and T. Shaw. 2013. Assessing the Impact of Latency and Jitter on the Perceived Quality of Call of Duty Modern Warfare 2. In Proceedings of HCI - Users and Contexts of Use. Springer-Verlag, Berlin, Heidelberg.

[2] G. Armitage. 2003. An Experimental Estimation of Latency Sensitivity in Multiplayer Quake 3. In Proceedings of IEEE ICON. Sydney, Australia.

[3] T. Beigbeder, R. Coughlan, C. Lusher, J. Plunkett, E. Agu, and M. Claypool. 2004 The Effects of Loss and Latency on User Performance in Unreal Tournament 2003. In Proceedings of ACM NetGames. Portland, OG, USA.

[4] M. Claypool, R. Eg, and K. Raaen. 2017. Modeling User Performance for Moving Target Selection with a Delayed Mouse. In Proceedings of Springer MMM. Reykjavik, Iceland.

[5] A. Depping and R. Mandryk. 2017. Why is This Happening to Me?: How Player Attribution Can Broaden Our Understanding of Player Experience. In Proceedings of ACM CHI. Denver, CO, USA.

[6] M. Dick, O. Wellnitz, and L Wolf. 2005. Analysis of Factors Affecting Players' Performance and Perception in Multiplayer Games. In Proceedings of ACM NetGames. Hawthorn, NY, USA.

[7] E\$ports Earning. 2019. Prize Money, Results, History, Statistics. Online: https: //www.esportsearnings.com/. (Accessed January 5, 2021).

[8] T. Fritsch, H. Ritter, and J. Schiller. 2005. The Effect of Latency and Network Limitations on MMORPGs: a Field Study of Everquest 2. In Proceedings of ACM NetGames. Hawthorne, NY, USA.

[9] C. Gough. 2020. eSports Audience Size Worldwide from 2018 to 2023. Statista. Online: https://tinyurl.com/y3tffxzo. (Accessed September 17, 2020).

[10] O. Hoßfeld, H. Fiedler, E. Pujol, and D. Guse. 2016. Insensitivity to Network Delay: Minecraft Gaming Experience of Casual Gamers. In Proceedings of the International Teletraffic Congress (ITC). IEEE, Würzburg, Germany.

[11] Z. Ivkovic, I. Stavness, C. Gutwin, and S. Sutcliffe. 2015. Quantifying and Mitigating the Negative Effects of Local Latencies on Aiming in 3d Shooter Games. In
Proceedings of ACM CHI. Seoul, Republic of Korea.

[12] W.K. Lee and R. Chang. 2015. Evaluation of Lag-Related Configurations in First-Person Shooter Games. In Proceedings of ACM NetGames. Zagreb, Croatia

[13] S. Liu, A Kuwahara, J. Scovell, J. Sherman, and M. Claypool. 2021. Lower is Better? The Effects of Local Latencies on Competitive First-Person Shooter Game Players. In Proceedings of ACM CHI. Yokohama, Japan, 12 pages.

[14] S. Liu, A. Kuwahara, J. Scovell, J. Sherman, and M. Claypool. 2021. The Effects of Network Latency on Competitive First-Person Shooter Game Players. In Proceedings of the 13th International Conference on Quality of Multimedia Experience (QoMEX). Virtual Conference.

[15] M. Long and C. Gutwin. 2018. Characterizing and Modeling the Effects of Local Latency on Game Performance and Experience. In Proceedings of ACM CHI Play. New York, NY, USA.

[16] M. Long and C. Gutwin. 2019. Effects of Local Latency on Game Pointing Devices and Game Pointing Tasks. In Proceedings of ACM CHI. Glasgow, Scotland, UK.

[17] K. Poels, Y.A.W. de Kort, and W.A. IJsselsteijn. 2007. D3.3 : Game Experience Questionnaire: Development of a Self-report Measure to Assess the Psychological Impact of Digital Games. Technische Universiteit Eindhoven.

[18] P. Quax, P. Monsieurs, W. Lamotte, D. De Vleeschauwer, and N. Degrande. 2004. Objective and Subjective Evaluation of the Influence of Small Amounts of Delay and Jitter on a Recent First Person Shooter Game. In Proceedings of ACM NetGames. Portland, OG, USA.

[19] Reuters.com. 2018. Investing in the Soaring Popularity of Gaming. Online: https://www.reuters.com/sponsored/article/popularity-of-gaming. (Accessed September 17, 2020).

[20] N. Sheldon, E. Girard, S. Borg, M. Claypool, and E. Agu. 2003. The Effect of Latency on User Performance in Warcraft III. In Proceedings of ACM NetGames. Redwood City, CA, USA.

[21] J. Spjut, B. Boudaoud, K. Binaee, J. Kim, A. Majercik, M. McGuire, D. Luebke, and J. Kim. 2019. Latency of $30 \mathrm{~ms}$ Benefits First Person Targeting Tasks More than Refresh Rate above $60 \mathrm{~Hz}$. In Proceedings of SIGGRAPH Asia. Brisbane, QLD, Australia. 